\title{
Sleep disorder diagnosis: the design and implications of online tools
}

\author{
Jacqueline Blake* and Don Kerr
}

\footnotetext{
* Correspondence: jblake@usc.edu.au School of Business, University of the Sunshine Coast, 90 Sippy Downs

Drive, Sippy Downs 4556, Australia
}

\begin{abstract}
Sleep disorders are a significant and growing problem, both for the economy of the nation and for the physical and psychological well-being of individual sufferers. The purpose of this research was to investigate the operational, administrative and medical environment within which sleep physicians currently diagnose patients with sleep disorders and develop an online support system that would more efficiently gather patient history data, and so improve the effectiveness of patient-physician consultations, the diagnoses, and patients' self-management of any subsequent treatment plans. The development of the online tools used a design science approach and those tools evaluated for usefulness and ease of use by physicians and the general population. The physicians found that the patient history information was in a form that facilitated assimilation with the removal of routine data collection allowing a more targeted or shorter consultation. The users found the application easy to use and that it made them think about their sleep quality. The implications for future practice of sleep disorder diagnosis and the value of the collection of patient history which would be available for analysis is also discussed.
\end{abstract}

Keywords: Health informatics; Chronic condition; Sleep disorders; Evidence base; Decision analytics

\section{Background}

Sleep disorders are a significant and growing problem, both for the economy of the nation and for the physical and psychological well-being of individual sufferers. Physicians, who deal with sleep disorders, and their administrative support staff and facilities, are under constant pressure to find more efficient ways of dealing with the backlog of patients, many of whom face significant wait times before being able to attend a consultation.

The main symptom of a sleep disorder is excessive daytime sleepiness there are a number of lifestyle consequences associated with this. Deloitte Access Economics (2010) state that $8.9 \%$ of the Australian population are affected. The direct and indirect costs of sleep disorders to the Australian society in 2010 was estimated to be 36.4 billion dollars (Deloitte Access Economics 2011). These indirect costs include comorbidities ${ }^{\mathrm{a}}$ such as hypertension, lost production, transport and workplace accidents and social costs, such as learning difficulties. Physically, sleep deprivation may be associated with an increased risk of myocardial infraction (heart attack), type two diabetes, and depressed immune response (Akerstedt and Nilsson 2003) and may present as

(c) 2014 Blake and Kerr; licensee Springer. This is an Open Access article distributed under the terms of the Creative Commons Attribution License (http://creativecommons.org/licenses/by/2.0), which permits unrestricted use, distribution, and reproduction in any medium, provided the original work is properly credited. 
depression. However treatment for sleep disorders reduces the direct and indirect costs restoring productivity and well-being to the individual and any organisation they are involved with. Sleep disorders are a chronic condition, so that for most conditions a treatment plan must be enduring and usually requires a considerable amount of ongoing commitment from the sufferer in partnership with their physician.

The estimate of $8.9 \%$ of the Australian population who have a sleep disorder is in all probability an underestimate with sleep disorders underdiagnosed in Australia (Deloitte Access Economics Deloitte Access Economics 2011), other countries report similar statistics (Young et al. 2002) with the people who are referred for treatment being strongly symptomatic (Kramer et al. 1999). This means that there remains a sector of the population who are yet to seek treatment and this is demonstrated by the direct costs of sleep disorders comprising only two per cent of the total cost. Sleep disorders commonly affect people around middle-age so as the population ages there are an increased number of people with sleep disorders. Risk factors in adults are obesity, alcohol consumption, smoking, nasal congestion and menopause (Young et al. 2002). The wait times for a consultation in a publically funded facility may be significant (Flemons et al. 2004). These wait times demonstrate a gap between demand for sleep disorder assistance and the ability of the most health services to provide for the current level of people referred to sleep clinics. Australia, in common with other developed countries is also facing an aging population in which sleep disorders will become more prevalent (Alzougool et al. 2008).

\section{Increasing demand for services}

This increasing service demand and a shortage of sleep disorder specialists (Australian Medical Workforce Advisory Committee 2000; Patlak 2005) have driven the sleep community interest in changing the traditional model of care for sleep disorders to one that leverages information systems to provide efficiencies and timely communication flows (Colten and Alterogt 2006). Abidi (2001) refers to the strategic use of knowledge derived from healthcare data as being "... pertinent towards the improvement of the operational efficiency of the said healthcare enterprise" (Abidi 2001, p. 6) while Hirakis and Karakounos (2006) and Haux (2010) state that knowledge management in healthcare allows the development of best practice models, making guidelines explicit and encourages innovation through development of the resultant knowledge base.

The term health informatics is used extensively throughout this paper, the term was developed by the International Medical Informatics Association (IMIA) and is defined as a combination of computer science, information science and health science designed to assist in the management and processing of data, information and knowledge to support healthcare and healthcare delivery (Conrick 2006, p. 4). Health informatics now forms part of the infrastructure for the delivery of healthcare with one of the major fields of research being decision analytics (Haux 2010). Health informatics allows the timely retrieval and filtering of patient and disorder information so that care givers have appropriate information in a quickly assimilated format within their work flow. Health informatics is the overarching domain within which this research lies as this research project aims to use decision analytics to assist in providing knowledge to support both the patient and physician in the diagnosis of sleep disorders. 


\section{Health and decision analytics}

One important avenue to leverage the capabilities of this project and its collection of sleep disorder data is that of business (or decision) analytics. Chen et al. (2012, p. 1166) defined business analytics as "techniques, technologies, systems, practices, methodologies and applications that analyse critical business data to help the enterprise better understand its business and market and make timely business decision". Davenport and Harris (2007) in their book titled "competing on analytics" suggest that business analytics offers companies a major competitive advantage and that companies that have higher returns on investment and usually companies with a higher level of analytical capabilities.

In the case we present here, we can consider a sleep clinic's business of being the most efficient and practical means of diagnosing and helping a patient deal with the lifestyle implications of a chronic disorder diagnosis. This has implications both in terms of using analytics to improve the sleep clinic's internal processes and thereby improving their return on investment and as a method of improving the capabilities (and in many cases, safety) of businesses across the entire economy of a nation, for example, having less sleepy commercial drivers in a transport company.

The purpose of this research was to investigate the operational, administrative and medical environment within which sleep physicians currently diagnose patients with sleep disorders and develop an online support system that would more efficiently gather patient history data. This allows us to improve the effectiveness of patientphysician consultations, the diagnoses, and patients' self-management of any subsequent treatment plans. The research used observations and a participatory research process based on inputs from two sleep physicians and a sleep psychologist to guide the development for the information technology artefact. This process ensured that the resultant online tools fitted into the physician's work flow with the patient information in the required format.

\section{Research objectives}

A design science approach was taken to building the online analytical tools. The resultant online tools incorporates four sub-applications,

1. An online sleep diary, with an output of a physician report showing a graphical visualisation of the patients sleep patterns and sleep statistics and the online presentation to the patient of the sleep pattern graph.

2. An online patient history questionnaire, asking lifestyle, medical history and sleep disorder specific questions.

3. A decision support system using input data from the patient history questionnaire.

4. A report generator analysing and presenting the output from the decision support system.

5. An evidence base of patient data available for data analytics.

These tools aim to remove responsibility for form filling from the physician to the patient, thus giving the patient access to sleep disorder specific questions earlier in their interaction with the sleep investigation unit. This will aid patient self-discovery of sleep 
disorder information that is relevant to their condition. Another outcome is the presentation of patient information to the physician within the natural workflow of a consultation and in a format which the physicians find useful.

One of the critical factors for physicians to accept a clinical decision support system is openness and transparency of how the decision was derived therefore an explanation of how a decision is reached by the system must be provided. The provision of an explanation module and the diagnostic environment of heterogeneous sleep disorder sufferers drove the adoption of small rule based decision support systems at every decision point in the argument toward diagnosis as described by Stranieri et al. (1999). Graber and Mathew (2008) found that to increase acceptance of clinical decision support systems (CDSS), reducing physician time to input data and fitting the system into physician work flow were key factors in the success of the CDSS.

\section{The IT artefact}

The IT artefact will aid physicians by presenting them with relevant patient information within the natural workflow of a consultation, and in a format that the physicians finds useful, while the individual who has a sleep disorder is able to fill in the questionnaire at their leisure and in their own home.

The development of an online application gives the form designer control over the sequence in which the form must be filled in, which means that the user can be constrained to provide information according to a required pattern. An online application also provides an opportunity to validate the entered data at the point of entry. Using validation in the sleep tool application means that the physician does not need to question the patient about the data contained in the application, but instead has time to talk about what the information means to the patient, for instance the impact of smoking on sleep disorders.

A well-developed sleep tool web application can be cost effective. The data sets from large numbers of respondents represent many repeated iterations of the form; consequently, as the data is captured, it can be automatically stored, manipulated and analysed. Thus data analysis is low cost and happens in real time for each form (Haynes et al. 2006). The automatic storage of collected information also provides an opportunity for good quality, validated data to be used for future research. This is important as at the moment no aggregated analysis is carried out on the present pen-and-paper patient history forms. Anecdotal evidence suggests that manually computing the data from a paper-based patient history, and the data entry of the information into a database, requires a large time commitment from clinic staff. This means that the routine collection of sleep diary information to build an evidence base is unlikely to be given a priority outside of a specific research project.

During 2009, there was strong growth in internet access in Queensland homes, reaching 87.6 per cent, which was an increase of $2 \frac{1}{2}$ per cent above the 2008 figure (Queensland Government Chief Information Office 2010). Minimal difference was shown in internet use between genders. The survey showed growth in computer access by people aged over 65; their internet use had grown strongly during the year to 61.1 per cent - a 6 per cent increase (Queensland Government Chief Information Office 2010). These percentages show that online forms are a viable alternative to paper-based forms for a large proportion of the population. 


\section{Method}

One of the difficulties in constructing an online information system is the need to design the application for unknown users. Users of an online system will have a range of technical skill levels, and will access the system through a variety of connection mechanisms, using an assortment of hardware. This diversity contrasts sharply with the development of an organisational system, which requires standardised connections and hardware, and whose operators have known, minimum technical standards (Taylor et al. 2003). Useability must be a primary objective of an online tool, Guenther (2004) stated that having a clear set of objectives for the user helps to make the online system a high value one. This project had two sets of users: physicians and patients. Consequently, the online tool had to meet two different sets of objectives and expectations. Calongne (2001) argues that what the user wants to achieve from the site must also be considered. In order to achieve user satisfaction, the online tools had to look and feel like a solid health orientated implementation. In the patients' case, the tool needed to feel like a device that would provide good information to them and to their physician, while in the physicians' case, the tool needed to provide a set of quality information in an easy to assimilate format.

To display information online, equity of access had to be considered, so that online tools had utility for the sight, hearing and physically impaired as well as users with little technical expertise. Wagner et al. (2010) suggest a number of guidelines for older users of web sites, including making sure visual elements are large enough to be seen and deciphered. They suggest using elements that contrast highly with the background, such as black elements on a white background. An uncluttered web page without too many elements claiming attention helps the user to make sense of the page. Also, graphics should be used only when necessary to illustrate or add to the site's function (Yen et al. 2007). For the online sleep tool application, the age range of the users was expected to be fairly broad and computer literacy to be variable. Most of the potential patients would be older than 45 and reasonably computer literate, but to improve equity of access the sleep diary had to be as accessible as possible across the whole spectrum of potential patients.

In the sleep tool application, the user interface was given the maximum contrast of black sans serif text on a plain white uncluttered background. The uncluttered background means that the sleep tool application can be personalised - with, for example, an organisation's logo - without disturbing the readability of the questions. The plain background also helps to minimise the file size of the web application, so that patients whose computers have slow download speeds are not penalised by having to wait for a graphic to appear.

The heuristic evaluation rules for websites detailed by Sharp et al. (2007) were employed. The website has internal consistency, with terms carrying the same meaning throughout the web site and the use of simple language. To aid internal consistency, formatting of pages, fonts, font sizes and font colours were made consistent. Shortcuts were not used, as the sleep tool application has a simple linear format which must be followed to gather complete information. The user's memory load is minimised with no information being required to be remembered from one part of the web-page or website to the other. Entries into the web form are validated; for example in the sleep diary, a wake time before a sleep time cannot be chosen, and throughout, each page is 
checked for completeness. The user is given feedback in two forms: for the sleep diary, the user is presented with a sleep pattern graphic that represents the entered sleep patterns; for the patient history questionnaire, the user receives a message that their information has been sent to their sleep clinic.

\section{Purpose of the web application}

One of the most important factors in the design of the sleep tool web application was the role the patient history played during a consultation. Physicians have clear expectations about what they consider to be best practice at this time, so patient history was included as an input to the system. In order to facilitate the knowledge acquisition process, the researcher formed a team with two sleep physicians and a psychologist. All three were considered to be experts in the field of sleep disorder diagnosis, and a collaborative approach was taken to help gain insights into their shared knowledge and experience in the sleep disorder domain and in order to develop the knowledge base for the DSS. All stakeholders (physicians and patients) provided approval for the process (as required by ethics). Gaining the physician stakeholders' approval of the output from the sleep tool application and its utility as a data gathering instrument for the patient history was essential. This approval meant the project gained access to the sleep physicians' patient knowledge and gave the medical team a vested interest in the success of the project (Cornwall and Jewkes 1995). The two sleep specialists provided expertise on the diagnostic criteria and process, while the psychologist specialised in psychological sleep disorders such as insomnia, the psychology impacts of sleep disorders and compliance with sleep disorder treatment by patients. The researcher acted as team leader to keep the team focused on the required outcome, researched questions from the team, set agendas, produced questionnaire drafts, scheduled meetings and kept records of the meetings.

\section{Structure of the web application}

The sleep tools application has three main areas of functionality (see Table 1).

There are four main categories of user in the sleep tools application (see Table 2).

The entry page of the online sleep application is a log-on page providing the facility to differentiate between different types of users and the means to authenticate users through the use of an identification number and user name. Differentiating between users is important to customise their interaction with the web site. To facilitate the physician's use of the tool the physician goes directly from log-on to the sleep statistic

Table 1 Sleep tools application main areas of functionality

\begin{tabular}{ll}
\hline Functionality & Output \\
\hline $\begin{array}{l}\text { An online sleep diary, which records 14 days } \\
\text { of a person's sleep habits and mood. }\end{array}$ & $\begin{array}{l}\text { Patient: a graphical representation of their sleep habits. } \\
\text { Physician: a graphical representation of a patient's sleep habits } \\
\text { and a report containing sleep statistics showing the averages } \\
\text { of sleep data. }\end{array}$ \\
An online patient history questionnaire. & Data stored in database. \\
A knowledge-based decision support system. & $\begin{array}{l}\text { Data retrieved from database, manipulated according to logic } \\
\text { embedded in decision support system and presented as a } \\
\text { report to the physician detailing if the person's patient history } \\
\text { meets the criteria for the main sleep disorders. }\end{array}$ \\
\hline
\end{tabular}


Table 2 Types of user in the web based sleep tools application

\begin{tabular}{ll}
\hline Type of user & Function \\
\hline A super-user or administrator & Limited to one user - has overall administration responsibility. \\
Hospital administration staff & $\begin{array}{l}\text { Add or edit patients and doctors. They may also access patient } \\
\text { results for their clinic, to print out results for patient files. }\end{array}$ \\
Physicians & Review and print their patients' results. \\
Patients & Complete sleep diary and patient history questionnaire. \\
\hline
\end{tabular}

page for the entered patient number, which allows for the entry of a user name and password. The next page has a different menu, depending on the class of user. The patient menu page allows the user to choose between either the sleep diary or the patient history areas of functionality or, alternatively, to return to the log-on page.

The main administrator or super-user menu shows that the main administrator can access all areas and has overall responsibility for administering the system.

A physician must be linked to a clinic in order for the physician menu screen to be created. This function can be completed by an administration officer (clinic administrator) or the super-user. A clinic administrator must also be associated with a clinic. Their role is to create patient records and support the physicians by printing patient reports. They may also create other administrator screens. These figures showing the management screens demonstrate the overlay of administration and security which controls the views that users have of the main areas of functionality.

\section{Online sleep diary}

A sleep diary is used to record sleep patterns, usually for fourteen nights. The person notes the details of their night's sleep day-by-day, building up a picture of sleep habits (known as sleep hygiene). Poor sleep hygiene, or insufficient time made available for sleep, may be a cause of excessive daytime sleepiness, the main symptom of a sleep disorder. Therefore, a sleep diary can be used to identify a cause for daytime sleepiness, lifestyle reasons or a sleep disorder. Sleep diaries are used by a number of health professionals, including psychologists, physicians and sleep specialists and is the primary diagnostic tool for identifying insomnia. It is a very useful aid in the diagnosis of other sleep disorders, as relatively short periods of time taken to fall asleep or frequent awakenings are indicators of poor quality sleep, and of sleep disorders.

During an exploratory interview early in a project to review sleep diagnosis, the remark was made by a director of a large public health clinic that ".. sleep diaries were a good tool which were not being used efficiently due to the timing and the short length of time available in the consultation to extract information from the data" (pers. Comm. 23 Sept.). The current format of the sleep diary used in the sleep investigation unit study was a pen-and-paper questionnaire in which the patient coloured in a square between two printed times - for example, nine and ten o'clock - to indicate bed time and waking up time.

\section{Data visualisation in the sleep diary}

Patient access to the information contained in an online sleep diary means the patient gains increased self-knowledge of their sleep hygiene, which has been shown to 
improve health outcomes (Smith et al. 2004). Patients should be able to self-monitor their sleep patterns. Wilde and Garvin (2007, p. 343) define self-monitoring as "awareness of symptoms or bodily sensations that is enhanced by periodic measurements recordings and observations to provide information for improved self-management". Self-monitoring of sleep patterns means that the patients have an opportunity for shared decision making with their physician on the management of their sleep disorder. Shared decision making is a significant factor in compliance with treatment plans (Sheppard et al. 2009). This is important in a chronic condition such as a sleep disorder, which has an ongoing treatment burden. A flow chart of this process is shown in Figure 1.

The daily results from the patient entry of sleep pattern data were built day by day into a graph shown in Figure 2. A graph was used to help patient's perform functional analysis of their sleep patterns, for instance does a caffeine containing drink before bed affect my sleep, am I allowing enough time for sufficient sleep or am I sleeping for a sufficient amount of time and still waking feeling unrefreshed. This graph is used to in Yau's (2011) words to tell the story of the data to both the patient and physician. The data visualisation tool is designed to assist patients to self-manage their chronic condition by making this data more accessible and therefore easier to assimilate. The traditional pen and paper sleep diary where the patient fills in specific times makes it difficult for the patient and physician to identify sleep patterns and areas of concern. For the physician, this visualisation provides a method where within a glance they can assimilate the patients sleep patterns, this is important as the consultation can then be patient centred rather than a general discussion on sleep patterns. This data visualisation demonstrates that these tools are useful to create models that have utility for single patients as well as large numbers of patients.

With this on-line system, the physician gains earlier access to patient sleep hygiene information and can therefore prioritise those patients who need to be seen first, ensuring that those in most need of the service will have the shortest wait times. It is also forecasted that patient satisfaction with the service will increase as during the wait time

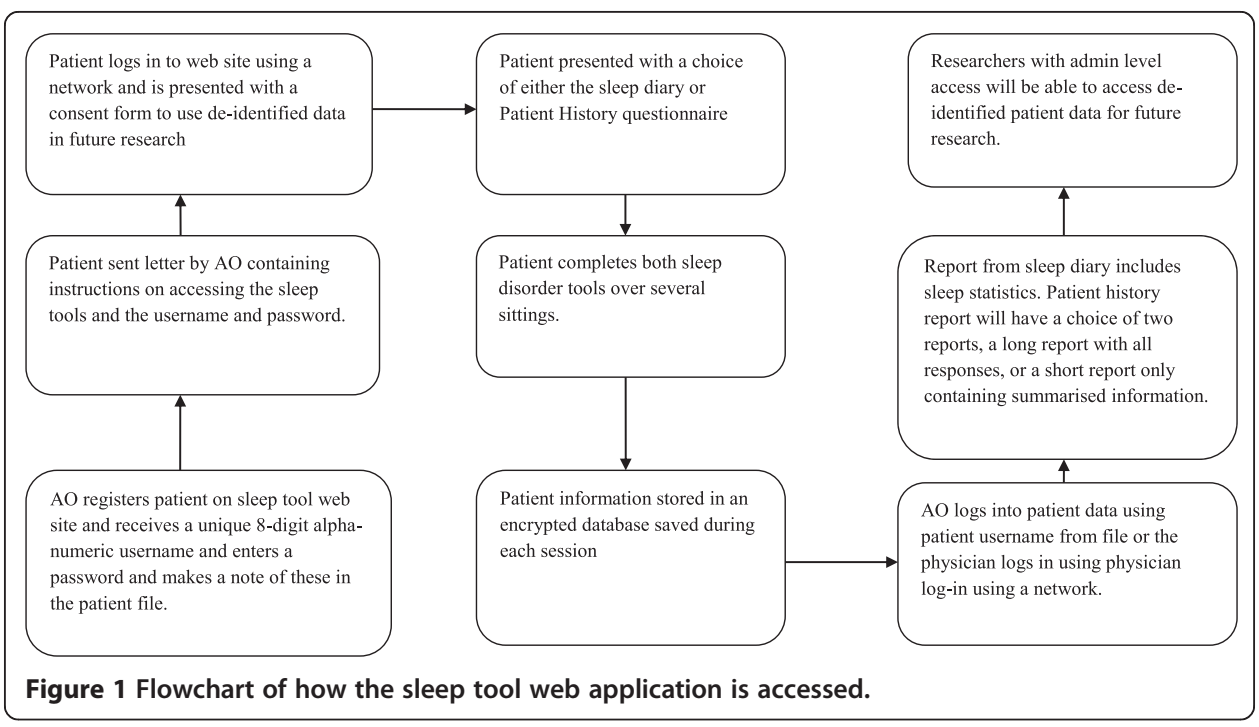




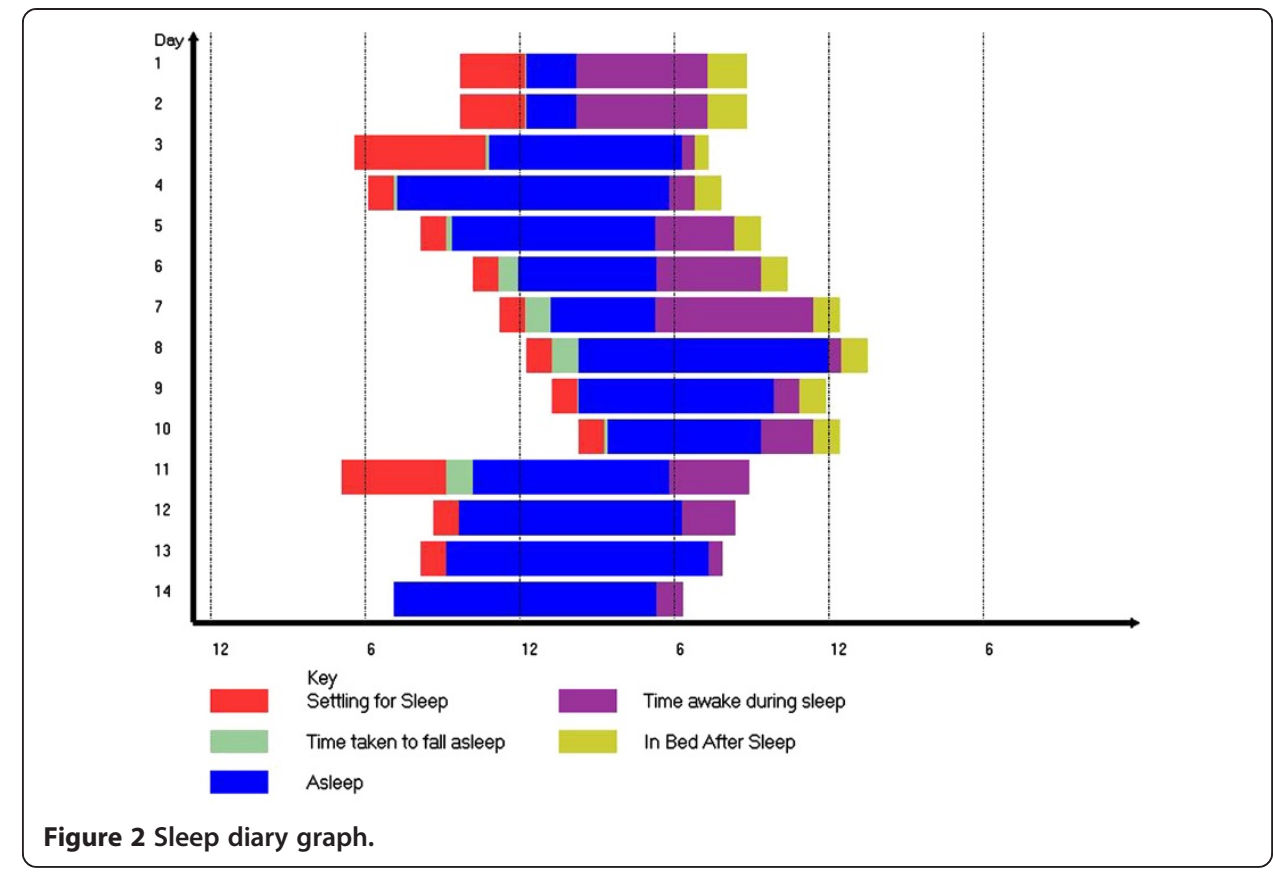

for an appointment they are able to self-monitor their condition. Therefore, the online sleep diary has a number of benefits, namely:

1. The data contained within the diary is presented in a summarised, readily accessible format for both physicians and patients.

2. The diary contents are available at any internet access point and in real time (subject to security precautions).

3. The diary facilitates the easy development of a database of sleep patterns and this can be used for historical data analysis.

4. The online diary has all the advantages of digital data such as reuse, multiple storage locations, easier transferability of data to other medical professionals and availability for statistical analysis.

Better quality sleep information is obtained when a sleep diary is filled out every day. This ensures that the person has the best recall of sleep quality and timing of sleep, Libman et al. (2000) stated that questionnaires that look at respective sleep patterns are susceptible to memory distortion, whereas a sleep diary filled in every day has the ability to capture day-to-day variations, highlighting variations that may indicate problems such as sleep deficits in sleep patterns. This means that the recording of a person's sleep habits over a two-week period in a sleep diary gives a baseline of a person's sleep patterns with greater precision than retrospective sleep quality questionnaires.

One disadvantage of a sleep diary is that subjects may change their sleep patterns in reaction to the self-monitoring and the imposition of filling in the diary day-today (Libman et al. 2000). However, Lacks and Morin (1992) comment that a sleep diary is the most widely used, practical and economical method of gauging sleep patterns. 
An online sleep diary has a number of benefits: the data contained within the diary are presented in a summarised, readily accessible format for both physicians and patients, and are available in real time, while the database of sleep patterns is being built.

\section{Online sleep diary data analysis and outputs}

The online sleep diary will produce a summary report which may be printed both for the person filling in the diary and for a physician. This summary report gives access to sleep statistics (see Table 3).

A count of the number of times that the patient reported waking up was checked, and the reasons given for the patient being kept awake was also reported. An average of the morning's mood and feeling are also given. The report is presented in a quickly assimilated format that has direct utility for assisting in a diagnosis of a sleep disorder. The summary report may also be added to a patient record in order to provide a baseline for future sleep diary reports for the physician and patient.

The facility to export the data contained in the online sleep diary in an eXtensible Markup Language (XML) format was also provided. This means that organisations other than that hosting the sleep diary can receive patient data in an electronic format, facilitating use of electronic record keeping.

\section{Current sleep diary model}

The current paper-based sleep diary, as stated above, does not present information in a format that the lay person can understand. The online sleep diary in this project presents sleep patterns as an easy to understand multi-coloured horizontal bar graph, with time along the $x$-axis. Midnight is the centre of the $x$-axis to allow the comparison of the regularity of bed and waking up times which are important in sleep hygiene. This graph builds day by day as the patient completes each day's diary entry. The different colours within the bars represent different states, such as awake during sleep time, sleep latency and sleep time. Therefore, the physician can gain a view of the patient's sleep patterns quickly, while the patient gains access to the information on their sleep patterns presented in a simple graphical format.

\section{Table 3 Sleep statistics in report for physician}

\begin{tabular}{ll}
\hline Name & Description \\
\hline Mean sleep duration. & Average time spent asleep. \\
Range of sleep duration. & $\begin{array}{l}\text { The minimum and maximum time spent asleep over the sleep diary time } \\
\text { period. }\end{array}$ \\
$\begin{array}{l}\text { Number of nights with fewer than } \\
\text { seven hours sleep. }\end{array}$ & $\begin{array}{l}\text { A count of the number of nights where fewer than seven hours of sleep } \\
\text { were achieved. }\end{array}$ \\
Mean for sleep onset. & $\begin{array}{l}\text { Average length of time taken between settling in bed ready for sleep; for } \\
\text { example, light off, television off, and time that sleep was achieved. }\end{array}$ \\
Range of sleep onset. & $\begin{array}{l}\text { The minimum and maximum times spent getting to sleep over the sleep } \\
\text { diary period. }\end{array}$ \\
Mean wake up time. & $\begin{array}{l}\text { Average time that the person wakes for the last time. } \\
\text { Sleep efficiency is the proportion of time spent asleep in the time } \\
\text { available for sleep; for example, of the nine hours spent in bed, eight } \\
\text { hours were spent asleep. The sleep efficiency is } 8 / 9=0.89 .\end{array}$ \\
\hline
\end{tabular}


The current model of a pen-and-paper sleep diary denies the patient an opportunity to self-monitor their sleep patterns and adopt functional analysis of lifestyle changes such as reducing caffeine intake. With the online sleep diary, patients gain a tool to use in self-monitoring their sleep patterns. Wilde and Garvin (2007, p.343) define selfmonitoring as awareness of symptoms that is facilitated by periodic measurements or recordings to provide information for improved self-management. The use of the online sleep diary to self-monitor gives patients the opportunity for shared decision making with their physician on the management of their sleep disorder. Vermeire and Hearnshaw (2001) found that shared decision making is an important factor in compliance to treatment.

The online sleep diary is a sub-application of the sleep tool web application, with the printed graph and sleep statistics providing a hard copy report for the physician.

\section{Development of the patient history questionnaire}

The patient history questionnaire is required to act as an information gathering point for input into the intelligent decision support system standard and a validated tool for patient appraisal in sleep disorder consultations. This will provide the sleep physician with good quality data before the first consultation with the patient. The responsibility for filling out the forms is transferred to the patient. This shift from the current practice will potentially free up physician time during the consultation and remove the need for the physician to ask information gathering closed-ended questions during the consultation; for example, "How many caffeine containing drinks did you consume during the day?" Instead, the physician will already have access to the patient's background information, so can use the limited consultation time to focus on the patient's sleep problem and to suggest lifestyle changes. This also has the potential to increase the patient's satisfaction with the provided service.

The outline of the proposed data gathering system for the decision support system for the diagnostic process was driven by a series of three one-hour interviews with the director of a Sleep Investigation Unit, Brisbane, Australia to discuss the sleep disorder diagnostic process. The convergent interviewing method was used to discover initial requirements. This informal interviewing technique is useful when the researchers are in some doubt about the information which is to be collected. Notes and impressions from the interviews were written in a journal and emailed to the interviewee for confirmation that these were aligned with the information they intended to convey. These meetings established the outline of the patient-physician interaction and discovered that there were two main types of consultations. The first was an exploratory consultation where the physician was investigating the current symptoms and patient history; the second was a shorter, confirmatory consultation where the physician discussed the diagnosis with the patient.

\section{Domain specialist knowledge acquisition}

The researcher formed a team with two sleep physicians and a psychologist. A collaborative approach was taken with the medical staff to gain their shared knowledge and experience in the sleep disorder domain as knowledge acquisition for the knowledge base of the decision support system. Gaining these stakeholders' approval of the 
instrument was essential to gain access to sleep investigation clinic patients so as to test the instrument, and to give these team members a vested interest in the success of the project (Cornwall and Jewkes 1995). The team members were two sleep specialists, a sleep psychologist; and the researcher. The two medical doctors provided expertise on the diagnostic criteria and process; the psychologist specialised in psychological sleep disorders such as insomnia, the psychological impacts of sleep disorders, and compliance with sleep disorder treatments by patients. The researcher led the team and in addition had domain experience in constructing questionnaires and providing information systems in knowledge management. The researcher was also responsible for keeping the team focused on producing a data gathering tool for the decision support system, for undertaking research activities outside the meeting, for checking the literature for parameters, and for confirming decisions.

\section{Results}

\section{Physician evaluation of sleep tools instrument}

Investigations confirmed that the physicians spend a large portion of the available consultation time on routine questions such as capturing details on smoking habits and caffeine consumption. One of the physician's initial comments on seeing a patient history report was that at least they could read it demonstrating how important how important information which is easy to assimilate is within the confines of a consultation.

The sleep tool web application is intended to be used first, by patients with potentially limited computer literacy, and then by physicians who will use the application for diagnostic purposes. It was therefore essential to ensure that the application was usable. The International Standards Organisation (ISO) defines usability in standard 9241-11 as "extent to which a product can be used by specified users to achieve specified goals effectively, efficiently and with satisfaction in a specified context of use" (Zikmund 2003). The online sleep tools were evaluated by sleep physicians, in terms of their perception of how the use of the tools would benefit their practice, the consultation and patient information. A sample of 267 people from a general population used the sleep tools and then completed a usability questionnaire, finally a technical expect evaluated the sleep tools in terms of their usability.

The physicians were sent ten outputs from the sleep tools from randomly selected sleep disorder patients who completed the sleep tools. This is equivalent to the patient information which the physician's will receive before a consultation when the proposed sleep tool consultation interaction is implemented. The physicians were then asked a set of questions focusing on any impact that receiving the information in this format and before a patient consultation would have of their practice. The operational impact of using the sleep tools was also investigated.

The physicians stated that the application was usable and useful, that it could improve their communication with their patients, and that it provided efficiencies during the patient-physician diagnostic interaction. Importantly use of the sleep tools did not impose a change of the physician's workflow except that they had access to good quality patient information before the patient consultation. There was still an opportunity for physician's to clarify patient information with the sleep tool report acting as an objective second opinion according to established international disorder criteria. 
For the validity of the sleep tools instrument, the ten outputs from sleep disorder people were considered by the sleep physicians. The physicians concurred that the patient outputs fell within the expected range of patient responses.

\section{Patient evaluation of sleep tools instrument}

A total of 267 users entered their information in the sleep tools web application and then completed a questionnaire developed by the researcher based upon the Technology Acceptance Model (TAM). The questions from this questionnaire were pretested on colleagues who had previously used TAM for evaluating applications (Soroush et al. 2010) The questionnaire focused upon the two different areas of functionality: the online sleep diary and the online patient history questionnaire. The usability questionnaire was completed when the person's interaction with the application was finished. The questionnaire was a short pen-and-paper document that was easy to fill in.

The 269 users who evaluated the sleep tools web application indicated that it is easy to use, easy to understand and easy to read. The results demonstrated that the interface of the sleep tools web application is suited to users with low computer literacy and can be considered relatively easy to read and understand. The users' responses indicated that filling in the application resulted in them thinking about and understanding the pattern of their sleeping habits indicating that this tool is usable for users over a range of ages, education and computer literacy. This agrees with Calongne (2001) who postulated in an opinion paper that usability should be a primary objective in web site design, suggesting that knowing the audience and their objective in using a web site should be considered to achieve usability. Guenther (2004) also agreed that for successful web site usability, one should focus on end user objectives in using the application This study also agrees with Williams (2000) that a simple clean design with high contrast between elements that stand out clearly against their background are important. This also agrees with the findings from the technical evaluation that the layout of the web application was in a simple linear layout. The layout of the application in a linear design also agreed with another of Guenther (2004) principles. Yen et al. (2007) in a review of literature and the proposal of a model for web design also agreed that a linear layout assisted user's navigation.

The user evaluation that the sleep tool web application was usable agrees with previous studies that the Internet administration of questionnaires offers a valid mode of delivery (Buchanan 2003; Carlbring et al. 2007; Johnson 2005). Buchanan (2003) reviewed the administration of three online medical questionnaires and found that with the exception of using norms gathered from online questionnaires to compare against those gathered in pen and paper questionnaires, online delivery of questionnaires was a valid delivery mode. Johnson (2005) found that for protocol validity, respondents to an online questionnaire were as consistent as those respondents using a pen and paper questionnaire. Carlbring et al. (2007) investigated the psychometric properties of online questionnaires in a test retest study using online and pen and paper questionnaires. The finding was that online administration of questionnaires was reliable and valid. The outcome from this study is that an online questionnaire offers a mode of delivery which respondents find comfortable and usable. 


\section{Technical evaluation of sleep tool instrument}

The technical evaluation revealed the sleep tools web application to be easy to use with a simple linear design. However, a number of usability issues were noted that were not identified by the patient usability research. Some of these issues, such as the minimalist observation, reflected the following of access considerations and a desire for regional patients with slower Internet access not to be penalised. This evaluation show that this desire for equity of access may impact on the feel of the instrument, and the input of a graphical user interface expert should be requested for the next iteration of the application.

\section{Discussion}

In this system the collection of routine patient information is done by the patient completing an online questionnaire. Due to the removal of data collection in a consultation, the physician can spend time with the patient discussing patient-specific symptoms and life-style. This will enable the physician to see more patients or they can perform a more in-depth consultation with each patient. The implication is that a shorter consultation will allow the sleep physician to see more patients and thereby lessening the consultation wait time. In this case a shorter consultation times maximises the use of physician time and represents a saving to the service provider while the patient still receives a good quality service.

The output from the sleep tools web application will allow an efficient, consistent triage process before seeing a patient for the first time. The operational implication of this is that a patient with a high risk of sleep apnoea can be sent to have a polysomnography before a consultation with a sleep physician. This acts to shorten the diagnostic interaction by at least one consultation freeing the physician to see more new patients and reducing the cost of treating each of these patients. As the sleep disorder clinic is government funded this offers a direct saving to the government health budget and will help to alleviate wait times to be seen for patients. The patient is also saved the burden of having to travel to one consultation. Dement (2008) suggests that an aging population will exacerbate that shortage finding time gains and efficiencies within the sleep disorder consultation interaction is important for patient wait times and the cost of treating the disorder.

One of the problems with the management of chronic conditions such as sleep disorders is to gain collaboration between health providers, such as sleep specialist and primary care physicians. The collection of electronic patient data allows an ease of patient information transfer. This means that the primary care physician is able to reinforce the need for life style changes suggested by the specialist to improve patient treatment.

\section{Managerial implications}

The sleep tools web application automatically saves the patient history and sleep diary data in a database. This database then forms a valuable resource for researchers in aggregated patient history data, with the facility to export unidentified data in an eXtensible Markup Language (XML) format. This aggregated information is not currently available within this health service as hard copy patient records are used. Another important feature of this system is that the evidence base will be build used a standardised 
data format, to aid in future data analytics (Koh and Tan 2011). One of the most important characteristics of the sleep disorder population is that it is heterogeneous, future use of decision analytics techniques and methodologies on the aggregated patient history data may lead to a discovery of a combination of factors which may be used to screen for individual sleep disorders in the wider population. This analytic approach has implications for screening of populations in whom an undetected sleep disorder poses a risk not only to themselves but also to other members of the community such as commercial drivers. The managerial implications of this, for organisations that employ commercial drivers, is that a low cost screening instrument would be available. This would lower the risks of undiagnosed drivers or workers having serious road accidents, or multiple small work accidents because of impaired cognitive function due to lack of sleep. For the sleep clinic having a low cost screening tool would mean an inflow of patients and increase in business as previously un-diagnosed people would now be referred for treatment.

Other managerial implications with respect to hospital management include anecdotal evidence that suggests that sleep laboratory time is available to perform more polysomnography tests. However, there are an insufficient number of appointment slots for a patient to see a sleep physician before undertaking a polysomnography sleep study. Consequently, the efficient use of a physician's time during a consultation is critical to maintaining the sleep unit's work flow and an appropriate wait time for patients before seeing a physician. Hence if they can reduce the amount of data which is considered critical for a diagnosis or assimilate data more quickly, they can reduce the amount of consultation time necessary.

The use of decision analytics would also help to produce screening instruments better targeted to sectors of the population such as woman. Currently there is no questionnaire that targets women for the prediction of obstructive sleep apnoea (Yukawa et al. 2009). There have been a number of studies (Chervin 2000; Shepertycky et al. 2005; Smith et al. 2002; Yukawa et al. 2009) showing that women present differently to a clinician with Obstructive Sleep Apnoea. Both the symptoms of depression and obstructive sleep apnoea can be described as sleepiness, fatigue, tiredness and lack of energy, so there is an overlap between these disorders however most clinicians expect extreme daytime sleepiness to be the overriding symptom of obstructive sleep apnoea (Chervin 2000). However the words that women use to describe their symptoms of obstructive sleep apnoea are more likely to be fatigue, tiredness and lack of energy leading primary care physicians to look for a diagnosis such as depression (Chervin 2000). Shepertycky et al. (2005) state that approximately $90 \%$ of women with moderate to severe obstructive sleep apnoea are undiagnosed or misdiagnosed leading to significant health impacts and costs to the health system. As obstructive sleep apnoea contributes to disorders such as hypertension, cardiovascular disease and depression, medical costs in patients before a diagnosis of obstructive sleep apnoea are higher than those in a control group as these comorbid conditions prove more resistant to treatment (Smith et al. 2002). Women who receive a diagnosis of depression before a subsequent diagnosis of obstructive sleep disorder are more likely to be treated by drugs for depression rather than psychoanalysis and may not suffer from depression at all. The managerial implications is that misdiagnosed woman with obstructive sleep apnoea will tend to have more time of work and have lower work performance than woman with a treated sleep disorder. 
Decision analytic techniques of the resulting evidence base from the sleep tools would also allow the sleep clinic to calculate the benefit of providing services such targeted lifestyle change activities including weight loss or psychoanalysis to aid in the treatment of conditions such as insomnia and patient empowerment (Chen et al. 2012).

\section{Conclusions}

The sleep tool objectives - that of devolving the responsibility for form filling from the physician to the patient, and giving the patient access to sleep disorder specific questions earlier in their interaction with the sleep investigation unit - have been met. This will aid patients in their self-discovery of their own sleep disorder information, as the literature shows that a more knowledgeable patient receives a more personalised consultation and is better able to comply with treatment plans. However the primary objective which was met was to improve the communication flows between the patient and physician. This objective meant the consultation could change from one where the patient was required to answer a series of questions to a consultation where there was time for a discussion on how a patient's condition might be best managed and fitted into their lifestyle. This type of discussion is important for treatment compliance for patients with a chronic condition such a sleep disorder. As sleep disorders commonly affect people from age 50 and on, the aging of populations make the efficient treatment of sleep disorders a critical concern of health organisations. The future value of the sleep tool application will be increased by the use of data analytic tools and techniques such as data visualization on the evidence base to provide a pathway to diagnosis and treatment efficiencies and new means of identifying sleep disorder sufferers. This approach lends itself to further, higher level analytical processes as the de-identified data is already in a digital format and can be analysed at a macro level to determine trends within categories of patients. In addition, longitudinal studies can be implemented to determine long term trends in this particularly problematic byu-product of modern human living.

\section{Endnote}

${ }^{\mathrm{a}}$ co-existing or additional diseases with reference to an initial diagnosis.

\section{Competing interests}

The authors declare that they have no competing interests.

\section{Authors' contributions}

JB and DK developed the conceptual framework for the literature review and IT Artefact. JB managed the review and appraised all included papers as part of her PhD studies, however DK also provided sections. JB carried out design and development of the application with the oversight of DK. Both authors contributed to the content, read and approved the final manuscript.

\section{Background papers}

This book chapter draws upon the following papers:

Blake, J \& Kerr, D 2010, 'Development of an online sleep diary for physician and patient use', Knowledge Management \& E-Learning: An International Journal, Special Issue on E-Health: Accessing Knowledge for Global Health, vol. 2, no. 2. Blake, J, Kerr, D \& Gururajan, R 2010, 'Development of knowledge management support for the sleep disorder diagnosis process', in Australian Conference on Information Systems: proceedings of theAustralian Conference on Information Systems Brisbane.

\section{Acknowledgements}

The authors wish to acknowledge the financial support from the Australian Research Council whose financial support made the project possible. The authors also wish to acknowledge the time and expertise supplied by Dr Douglas, Dr Sellers and Dr Smith. 
Received: 20 November 2013 Accepted: 13 February 2014

Published: 7 March 2014

\section{References}

Abidi, SSR. (2001). Knowledge management in healthcare: towards 'knowledge-driven' decision-support services. International Journal of Medical Informatics, 63(1-2), 5-18. Science direct.

Akerstedt, T, \& Nilsson, PM. (2003). Sleep as restitution: an introduction. Journal of Internal Medicine, 254(1), 6-12.

Alzougool, B, Chang, S, \& Gray, K. (2008). Towards a comprehensive understanding of health information needs. Electronic Journal of Health Informatics, 3(2), e15.

Australian Medical Workforce Advisory Committee. (2000). The Specialist Thoracic Medicine Workforce. Australia: AMWAC Report. viewed 31 March 2008, https://www.hwa.gov.au/sites/uploads/The\%20specialist\%20thoracic\%20medicine\% 20workforce\%20in\%20Australia.pdf.

Buchanan, T. (2003). Internet-based questionnaire assessment: Appropriate use in clinical contexts. Cognitive Behaviour Therapy, 32(3), 100-109. Ebscohost - Academic Search Premier.

Calongne, CM. (2001). Designing for web site usability. Journal of Computing Sciences in Colleges, 16(3), 39-45. ACM Digital Library.

Carlbring, P, Brunt, S, Bohman, S, Austin, D, Richards, J, Öst, L-G, \& Andersson, G. (2007). Internet vs. paper and pencil administration of questionnaires commonly used in panic/agoraphobia research. Computers in Human Behavior, 23(3), 1421-1434.

Chen, H, Chiang, RHL, \& Storey, VC. (2012). Business intelligence and analytics: From big data to big impact. MIS Quarterly, 36(4), 1165-1188.

Chervin, RD. (2000). Sleepiness, fatigue, tiredness, and lack of energy in obstructive sleep apnea. Chest, 118(2), 372-379.

Colten, HR, \& Alterogt, MM. (2006). Sleep disorders and sleep deprivation: An unmet public health problem. National Academies Press. viewed 29/12/2008, http://octane.serveftp.com:8084/Classificados\%20na0\%20guardados/Biology/ Neuroscience/Sleep\%20Disorders\%20and\%20Sleep\%20Deprivation\%20-\%20H.\%20Colten,\%20B.\%20Altevogt\%20 (NAP,\%202006)\%20WW.pdf.

Conrick, M. (2006). Health informatics: transforming healthcare with technology. Melbourne: Thomson social science press.

Cornwall, A, \& Jewkes, R. (1995). What is participatory research? Social Science and Medicine, 41(12), 1667-1676.

Davenport, TH, \& Harris, JG. (2007). Competing on Analytics: the new science of winning. United States of America: Harvard Business School.

Deloitte Access Economics. (2011). Re-awakening Australia: The economic cost of sleep disorders in Australia, 2010. Australia: Sleep Health Foundation.

Dement, WC. (2008). History of sleep medicine. Sleep Medicine Clinics, 3, 147-156.

Flemons, WW, Douglas, NJ, Kuna, ST, Rodenstein, DO, \& Wheatley, J. (2004). Access to diagnosis and treatment of patients with suspected sleep apnea. American Journal of Respiratory and Critical Care Medicine, 169, 668-672.

Graber, ML, \& Mathew, A. (2008). Performance of a web-based clinical diagnosis support system for internists. Journal of General Internal Medicine, 23(Supplement 1), 37-40.

Guenther, K. (2004). Know the fundamentals and good design will follow. Online, 28(1), 54-56.

Haux, R. (2010). Medical informatics: Past, present, future. International Journal of Medical Informatics, 79(9), 599-610. Sciencedirect.

Haynes, RB, Sackett, DL, Guyatt, GH, \& Tugwell, P. (2006). Clinical epidemiology: how to do clinical practice research (3rd ed.). United States of America: Lippincott Williams \& Wilkins.

Hirakis, O, \& Karakounos, S. (2006). Goals and benefits of knowledge management in healthcare. In AA Lazakidou (Ed.), Handbook of Research on Informatics in Healthcare and Biomedicine, Idea Group Reference (pp. 193-200).

Johnson, JA. (2005). Ascertaining the validity of individual protocols from Web-based personality inventories. Journal of Research in Personality, 39(1), 103-129. ScienceDirect.

Koh, HC, \& Tan, G. (2011). Data mining applications in healthcare. Journal of Healthcare Information Management, 19(2), 65.

Kramer, NR, Cook, TE, Carlisle, CC, Corwin, RW, \& Millman, RP. (1999). The role of the primary care physician in recognizing obstructive sleep apnea. Archives of Internal Medicine, 159(9), 965-968.

Lacks, P, \& Morin, CM. (1992). Recent advances in the assessment and treatment of insomnia. Journal of Consulting and Clinical Psychology, 60(4), 586-594.

Libman, E, Fichten, CS, Bailes, S, \& Amsel, R. (2000). Sleep questionnaire versus sleep diary: which measure is better? International Journal of Rehabilitation and Health, 5(3), 205-209.

Patlak, M. (2005). Tips to help you detect common sleep disorders, American College of Physicians. viewed 31 March 2008, http://www.acpinternist.org/archives/2005/03/sleep.htm.

Queensland Government Chief Information Office. (2010). 2009 Queensland Household Survey - Computer and Internet Use. viewed 7 February 2011, http://www.abs.gov.au/AUSSTATS/abs@.nsf/Lookup/1318.3Feature\%20Article14Mar\% 202010.

Sharp, H, Rogers, Y, \& Preece, J. (2007). Interaction design: beyond human-computer interaction (2nd ed.). Chichester: John Wiley and Sons Limited.

Shepertycky, MR, Banno, K, \& Kryger, MH. (2005). Differences between men and women in the clinical presentation of patients diagnosed with obstructive sleep apnea syndrome. Sleep, 28(3), 309-314.

Sheppard, VB, Williams, KP, Harrison, TM, Jennings, Y, Lucas, W, Stephen, J, Robinson, D, Mandelblatt, JS, \& Taylor, KL. (2009). Development of decision-support intervention for black women with breast cancer. Psychooncology(9999), 62-70. viewed 01 September 2009, Wiley InterScience.

Smith, AE, Nugent, CD, \& McClean, SI. (2002). Implementation of intelligent decision support systems in health care. Journal of Management in Medicine, 6(2), 206-218.

Smith, S, Lang, C, Sullivan, K, \& Warren, J. (2004). Two new tools for assessing patients' knowledge and beliefs about obstructive sleep apnea and continuous positive airway pressure therapy. Sleep Medicine, 5(4), 359-367.

Soroush, L, Hafeez-Baig, A, \& Gururajan, R. (2010). Clinicians' perception of using digital stethoscopes in telehealth platform: Queensland telehealth preliminary study. Brisbane. https://eprints.usq.edu.au/19946/. 
Stranieri, A, Zeleznikow, J, Gawler, M, \& Lewis, B. (1999). A hybrid rule - neural approach for the automation of legal reasoning in the discretionary domain of family law in Australia. Artifical Intelligence and Law, 7(2-3), 153-183. Springerlink.

Taylor, MJ, Wade, S, \& England, D. (2003). Informing IT system web site design through normalisation. Internet Research: Electronic Networking Applications and Policy, 13(5), 342-355. Emerald.

Vermeire, E, Hearnshaw, H, Van Royen, P, \& Denekens, J. (2001). Patient adherence to treatment: three decades of research. A comprehensive review. Journal of Clinical Pharmacy and Therapeutics, 26, 331-342.

Wagner, N, Hassanein, K, \& Head, M. (2010). Computer use by older adults: a multi-disciplinary review. Computers in Human Behavior, 26(5), 870-882. ScienceDirect.

Wilde, MH, \& Garvin, S. (2007). A concept analysis of self-management. Journal of Advanced Nursing, 57(3), 339-350. Wiley Interscience.

Williams, TR. (2000). Guidelines for designing and evaluating the display of information on the web. Technical Communication, 47(3), 383-397.

Yau, N. (2011). Visualize This: The flowing data guide to design, visualization, and statistics. Indianapolis: John Wiley \& Sons.

Yen, B, Hu, PJ-H, \& Wang, M. (2007). Toward an analytical approach for effective Web site design: a framework for modeling, evaluation and enhancement. Electronic Commerce Research and Applications, 6(2), 159-170. ScienceDirect.

Young, T, Peppard, PE, \& Gottlieb, DJ. (2002). Epidemiology of obstructive sleep apnea: a population health perspective. American Journal of Respiratory and Critical Care Medicine, 165(9), 1217-1239.

Yukawa, K, Inoue, Y, Yagyu, H, Hasegawa, T, Komada, Y, Namba, K, Nagai, N, Nemoto, S, Sano, E, Shibusawa, M, Nagano, N, \& Suzuki, M. (2009). Gender Differences in the clinical characteristics among Japanese patients with obstructive sleep apnea syndrome. Chest, 135(2), 337-343.

Zikmund, WG. (2003). Business research methods (7th ed.). Mason United States of America: Thomson South-Western.

doi:10.1186/2193-8636-1-7

Cite this article as: Blake and Kerr: Sleep disorder diagnosis: the design and implications of online tools. Decision Analytics 2014 1:7.

\section{Submit your manuscript to a SpringerOpen ${ }^{\circ}$ journal and benefit from:}

- Convenient online submission

Rigorous peer review

- Immediate publication on acceptance

- Open access: articles freely available online

- High visibility within the field

- Retaining the copyright to your article

Submit your next manuscript at $\boldsymbol{\nabla}$ springeropen.com 